Влияние диуретической терапии на показатели сигнал-усредненной ЭКГ предсердного комплекса и наджелудочковые аритмии у больных ишемической болезнью сердца с хронической сердечной недостаточностью

\author{
Х.Х. Шугушев*, А.А. Гаева
}

Кабардино-Балкарский государственный университет им Х.М.Бербекова

\title{
Diuretic therapy effects on the signal-averaged ECG parameters of atrial complex and supraventricular arrhythmias in patients with coronary heart disease and chronic heart failure
}

Kh.Kh. Shugushev*, A.A. Gaeva

Kh.M. Berbekov Kabardino-Balkar State University

Цель. Изучить показатели сигнал-усредненной (СУ) ЭКГ предсердного комплекса и частоты наджелудочковых аритмий (НЖА) у больных ишемической болезнью сердца (ИБС) с хронической сердечной недостаточностью $(\mathrm{XCH})$ на фоне терапии диуретиками (Д) торасемидом и фуросемидом.

Материал и методы. Обследованы 107 больных ИБС с ХСН. Всем больным проводили 24-часовое мониторирование ЭКГ и регистрацию СУ ЭКГ предсердного комплекса в динамике. 52 больных в качестве терапии Д получали фуросемид, 55 - торасемид.

Результаты. На фоне терапии Д в обеих группах (гр.) отмечено некоторое увеличение длительности фильтрованного P и LAS5, а также снижение значения RMS20. Указанные изменения были максимально выраженными у пациентов, лечившихся фуросемидом. Сравнение динамики различных НЖА при терапии Д демонстрирует менее выраженное негативное влияние торасемида: в этой гр. отмечено снижение количества одиночных, парных и групповых наджелудочковых экстрасистол.

Заключение. Больные ИБС с ХСН характеризуются нарушением процессов деполяризации предсердий, которые проявляются неблагоприятными изменениями показателей СУ ЭКГ предсердного комплекса. Терапия торасемидом оказывает менее выраженное отрицательное влияние на показатели СУ ЭКГ предсердного комплекса и сопровождается снижением частоты НЖА, что делает этот препарат более предпочтительным для лечения таких больных.

Ключевые слова: ишемическая болезнь, хроническая сердечная недостаточность, диуретическая терапия, сигнал-усредненная ЭКГ.

Aim. To study the signal-averaged ECG (SA-ECG) parameters of atrial complex and supraventricular arrhythmias (SVA) in patients with coronary heart disease (CHD) and chronic heart failure (CHF), treated with diuretics (D) torasemide and furosemide.

Material and methods. The study included 107 patients with CHD and CHF. All participants underwent 24-hour ECG monitoring and the registration of SA-ECG atrial complex parameters. In total, 52 patients received furosemide, and 55 were administered torasemide.

Results. In both groups, D therapy was associated with some increase in the duration of filtered P and LAS5, as well as a decrease in RMS20. These changes were more pronounced in the furosemide-treated patients. Torasemide demonstrated better effects on the SVA dynamics, which manifested in the decreased numbers of single, paired, and grouped SV extrasystoles.

Conclusion. In CHD and CHF patients, the disturbed atrial depolarisation was manifested in negative dynamics of SA-ECG atrial complex parameters. Torasemide therapy did not affect the atrial complex parameters and also reduced SVA incidence, which made torasemide a medication of choice for patients with CHD and CHF.

Key words: Coronary heart disease, chronic heart failure, diuretic therapy, signal-averaged ECG.

(с)Коллектив авторов, 2011

e-mail goster@kbsu.ru

[Шугушев Х.Х. (*контактное лицо) - заведующий кафедрой госпитальной терапии медицинского факультета, Гаева А.А. - аспирант кафедры]. 
Ишемическая болезнь сердца (ИБС) - широко распространенное заболевание сердечно-сосудистой системы (ССС). Хроническая сердечная недостаточность $(\mathrm{XCH})-$ одно из частых и прогностически неблагоприятных осложнений ИБС. Основным клиническим проявлением ХСН является отечный синдром, в связи с чем диуретическая терапия - важное звено в лечении больных с ХCH. При этом лечение диуретиками (Д) связано с нарушением электролитного баланса, неблагоприятным влиянием на нейрогормональные системы. Клиническими проявлениями таких нарушений может стать усиление электрической нестабильности миокарда, различные аритмии и внезапная сердечная смерть (ВСС) [1,2]. В то же время неинвазивная оценка электрической нестабильности миокарда широко применяется в настоящее время. Впервые, используя сигнал-усредненную электрокардиограмму (СУ ЭКГ), было выявлено нарушение деполяризации предсердий у больных с пароксизмами фибрилляции предсердий (ФП) [3]. По данным литературы, поздние потенциалы предсердий (ППП) регистрируют у 63-93 \% больных с ФП. Наличие ППП считают маркером высокого риска развития наджелудочковых аритмий (НЖА) и ФП [4-6]. В настоящее время отсутствует единый подход к определению ППП, а клиническая оценка показателей СУ ЭКГ предсердного комплекса неоднозначна. Влияние же лекарственной терапии, в частности, Д, на эти показатели и их взаимосвязь с НЖА практически не изучено.

Цель работы - изучение показателей СУ ЭКГ предсердного комплекса и частоты НЖА у больных ИБС с ХСН на фоне терапии Д - торасемидом и фуросемидом.

\section{Материал и методы}

В исследование были включены 107 пациентов:

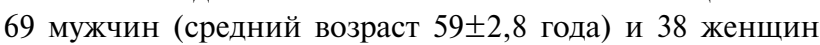
(средний возраст $65 \pm 4,3$ года), находившихся на лечении в ГУЗ “Кардиологический центр” Кабардино-Балкарской Республики. У 26 больных диагностирована стабильная стенокардия III функционального класса (ФК) по классификации Канадской ассоциации кардиологов, у 23 - IV ФК, 58 пациентов перенесли инфаркт миокарда (ИМ), у 66 регистрировалась артериальная гипертония (АГ). У 60 больных диагностирована IIA стадия (стд.) ХCH, у 47 больных - ІІБ стд. До начала терапии Д 84 пациента имели III ФК, 23 - IV ФК ХСН согласно классификации Нью-йоркской ассоциации сердца (NYHA).

Больных с острым коронарным синдромом (ОКС), эндокринными, инфекционными и нервно-психическими заболеваниями в исследование не включали. Для сравнения исходных показателей СУ ЭКГ предсердного комплекса и НЖА использовалась контрольная группа (ГК), которую составили 30 практически здоровых людей, сопоставимых с группой (гр.) больных - основная группа (ОГ), по возрастному и половому признакам.

Всем больным проводили клиническое обследование с оценкой жалоб, объективного состояния, результатов лабораторных и инструментальных исследований: электрокардиография (ЭКГ), эхокардиография (ЭхоКГ), тест 6-минутной ходьбы (т6мх), 24-часовое мониторирование (CM) ЭКГ до и через 10 сут. после начала терапии Д. СУ ЭКГ регистрировали с помощью компьютерного электрокардиографа KARDi (MKC, Россия) по описанной ранее общепринятой методике [7]. Критериями обнаружения ППП считали продолжительность фильтрованного P > 125 мс, продолжительность LAS5 > 20 мс, амплитуда RMS20 < 3,5 мкВ [8].

Больные с ХCH были рандомизированы методом случайных чисел на 2 гр.: 52 пациента I гр. в качестве петлевого Д (пД) получали фуросемид в дозе 20-60 мг/ сут., 55 пациентов II гр. - торасемид (Диувер, TEVA, Израиль) в дозе 5-20 мг/сут. . Увеличение дозы Д проводили ежедневно (фуросемид на 20 мг, торасемид на 5 мг) до достижения адекватного диуреза.

Все больные получали терапию ингибиторами ангиотензин-превращающего фермента (ИАПФ) и $\beta$-адреноблокаторами ( $\beta$-АБ). В гр. фуросемида 27 $(51,9 \%)$ больных получали бисопролол (2,5-5 мг/сут.), 20 $(38,5 \%)$ больных - карведилол (12,5-25 мг/сут.), 5 (9,6 \%) больных - небиволол (2,5-5 мг/сут.). В гр. торасемида аналогичные $\beta$-АБ получали 28 (50,9\%), 19 (34,5\%) и 8 $(14,5 \%)$ больных, соответственно. В гр. фуросемида 15 $(28,8 \%)$ больных получали квинаприл (10-20 мг/сут.), 18 (34,6\%) больных - зофеноприл (15-30 мг/сут.), 19 (36,5\%) больных - периндоприл (2,5-10 мг/сут.). В гр. торасемида аналогичные ИАПФ получали 20 (36,4 \%), 15 (27,3\%) и 20 $(36,4 \%)$ больных, соответственно. Таким образом, обследуемые гр. больных не отличались по фоновой терапии.

Протокол исследования был одобрен этическим комитетом. Пациенты, участвующие в исследовании, были ознакомлены с целью и дизайном исследования и подписали информированное согласие на участие в исследовании.

Для оценки значений параметров использовали средние значения и их стандартные ошибки $(\mathrm{M} \pm \mathrm{m})$. Достоверность различий средних значений между гр. оценивалась с помощью непарного -критерия Стьюдента, достоверность динамики средних значений в гр. - с помощью парного t-критерия Стьюдента. Достоверность различий в частоте выявления клинических признаков оценивали с помощью критерия $\chi^{2}$ Пирсона.

\section{Результаты и обсуждение}

Длительность фильтрованного Р, низкоамплитудных сигналов LAS5, среднеквадратичная амплитуда последних 20 мс фильтрованного сигнала QRS RMS20, а также доля пациентов с наличием ППП в обследованных гр. до начала терапии Д достоверно не различались. При этом отмечается их существенные отличия от аналогичных показателей ГК (таблица 1).

В литературе есть работы, посвященные изучению показателей СУ ЭКГ предсердного комплекса у больных различными сердечно-сосудистыми заболеваниями (СС3). Параметры СУ ЭКГ предсердного комплекса максимально изменены у больных ИБС, достоверно отличаясь от таковых у лиц ГК [9]. На фоне 10-суточной терапии Д отмечена практически одинаковая положительная динамика клини- 
Динамика показателей СУ ЭКГ предсердного комплекса больных ИБС с ХСН

Таблица 1 на фоне лечения фуросемидом и торасемидом

\begin{tabular}{llllll}
\hline Показатели & ГК & \multicolumn{2}{l}{ Гр. фуросемида $(\mathrm{n}=52)$} & \multicolumn{2}{l}{ Гр. торасемида $(\mathrm{n}=55)$} \\
\cline { 3 - 6 } & $(\mathrm{n}=30)$ & До лечения & На фоне лечения & До лечения & На фоне лечения \\
\hline Pfi, мc & $85,4 \pm 4,8$ & $111,3 \pm 1,33 \sim$ & $121,8 \pm 2,0 \sim *$ & $112,3 \pm 1,19 \sim$ & $114,0 \pm 1,27 \sim^{\wedge}$ \\
LAS5, мс & $12,7 \pm 2,7$ & $15,2 \pm 0,68$ & $17,4 \pm 1,4^{*}$ & $15,6 \pm 0,93$ & $15,9 \pm 0,81$ \\
RMS20, мкв & $37,8 \pm 4,1$ & $5,4 \pm 0,42 \sim$ & $3,59 \pm 0,43 \sim *$ & $5,6 \pm 0,7 \sim$ & $4,2 \pm 0,8 \sim$ \\
ППП, $\mathrm{n}(\%)$ & $1(3 \%)$ & $9(17,3 \%)$ & $15(28,8 \%)$ & $10(18,2 \%)$ & $9(16,4 \%)$ \\
\hline
\end{tabular}

Примечание: $-\mathrm{p}<0,05$ по сравнению с ГК; ${ }^{*}-\mathrm{p}<0,05$ по сравнению с аналогичным показателем до лечения; ${ }^{\wedge}-\mathrm{p}<0,05$ по сравнению с гр. фуросемида.

Таблица 2

Количество НЖА у больных с ХСН на фоне терапии фуросемидом и торасемидом

\begin{tabular}{llllll}
\hline Показатель & \multirow{2}{*}{$\begin{array}{l}\text { ГК } \\
(\mathrm{n}=30)\end{array}$} & \multicolumn{2}{l}{ Фуросемид $(\mathrm{n}=52)$} & Торасемид $(\mathrm{n}=55)$ \\
\cline { 3 - 6 } & & Исходные данные & После лечения & Исходные данные & После лечения \\
\hline Одиночная НЖЭ, в сут. & $138,9 \pm 42,4$ & $1083,6 \pm 826,5 \sim$ & $1089,9 \pm 744,1$ & $1078,7 \pm 957,3 \sim$ & $277,8 \pm 193,6^{* \wedge}$ \\
Парная НЖЭ, в сут. & $1,7 \pm 1,3$ & $101,6 \pm 6,26 \sim$ & $103,9 \pm 50,6$ & $184,1 \pm 179,2 \sim$ & $36,0 \pm 34,7^{* \wedge}$ \\
Групповая НЖЭ, в сут. & & $0,76 \pm 0,41$ & $12,4 \pm 6,6^{*}$ & $44,1 \pm 43,5$ & $5,4 \pm 4,1^{* \wedge}$ \\
НЖТ, в сут. & & $0,3 \pm 0,23$ & $1,1 \pm 0,6$ & $0,2 \pm 0,1$ & $0,2 \pm 0,2$ \\
\hline
\end{tabular}

Примечание: ${ }^{\sim}-\mathrm{p}<0,05$ по сравнению с ГK; * $-\mathrm{p}<0,05$ по сравнению с аналогичным показателем до лечения; ${ }^{\wedge}-\mathrm{p}<0,05$ по сравнению с гр. фуросемида.

Коррелятивные связи различных НЖА и показателей СУ ЭКГ предсердного комплекса

\begin{tabular}{llll}
\hline Показатели & Pf & LAS5 & RMS20 \\
\hline Одиночные НЖЭ & 0,25 & 0,15 & $-0,16$ \\
Парные НЖЭ & $\mathrm{p}=0,065$ & $\mathrm{p}=0,27$ & $\mathrm{p}=0,24$ \\
& 0,13 & 0,10 & $-0,20$ \\
Групповые НЖЭ & $\mathrm{p}=0,35$ & $\mathrm{p}=0,47$ & $\mathrm{p}=0,15$ \\
& 0,12 & 0,05 & $-0,17$ \\
Пароксизмы НЖТ & $\mathrm{p}=0,37$ & $\mathrm{p}=0,73$ & $\mathrm{p}=0,02$ \\
& 0,43 & 0,05 & $-0,31$ \\
\hline
\end{tabular}

ческих показателей в обеих гр. Уменьшение одышки наблюдалось у 50 (91\%) больных в гр. торасемида и у 48 (92\%) больных в гр. фуросемида; уменьшение отеков нижних конечностей - у 26 (47\%) и 25 (48 \%) больных; застойных явлений в малом круге кровообращения - у $35(64 \%)$ и $34(65 \%)$; уменьшение размеров печени - у 20 (36 \%) и 18 (35\%) больных, соответственно.

На фоне терапии Д в обеих гр. отмечено некоторое увеличение длительности фильтрованного Р и LAS5, а также снижение значения RMS20. Указанные изменения были максимально выраженными у пациентов, получавших фуросемид: произошло достоверное увеличение длительности фильтрованного Р, низкоамплитудных сигналов LAS5 и снижение квадрата амплитуды RMS20. При этом количество пациентов с критериями наличия ППП увеличилось до 28,8 \%. У пациентов, принимавших торасемид, неблагоприятные изменения показателей СУ ЭКГ предсердного комплекса были минимально выражены, а их изменение - статистически недостоверно. В то же время количество больных с ППП несколько сократилось, составив $16,4 \%$.
Выявленные различия значений Р, LAS5 и RMS20 на фоне терапии свидетельствуют об отсутствии выраженного неблагоприятного влияния торасемида на показатели ЭКГ высокого разрешения предсердного комплекса.

В таблице 2 представлена динамика частоты НЖА сердца по результатам 24-часового мониторирования ЭКГ.

Исходная частота нарушений ритма сердца у больных с ХСН отличалась от ГК. У пациентов с ХCH отмечена более высокая частота обнаружения различных НЖА, в т.ч., пароксизмов наджелудочковой тахикардии (НЖТ). Сравнение динамики различных НЖА при терапии Д демонстрирует менее выраженное негативное влияние на них торасемида: в этой гр. отмечено снижение количества одиночных, парных и групповых наджелудочковых экстрасистол (НЖЭ).

Указанную динамику частоты НЖА у пациентов, получавших фуросемид, можно объяснить как возможными электролитными нарушениями, так и негативным влиянием на вегетативную регуляцию ритма сердца. Уменьшение количества НЖА у пациентов, получавших торасемид, может быть связано 
с отсутствием его неблагоприятного влияния на электролитный состав, а также с улучшением показателей СУ ЭКГ предсердного комплекса.

При количественной оценке степени взаимосвязи между показателями СУ ЭКГ предсердного комплекса и НЖА обнаружены сильные коррелятивные связи между частотой пароксизмальной НЖТ и длительностью фильтрованного P ( $\mathrm{r}=0,44$, $\mathrm{p}<0,001)$, а также квадратом амплитуды RMS20 (r=$0,31, \mathrm{p}<0,05)$.

Значения длительности фильтрованного Р $>145$ мс и квадрата амплитуды RMS20 < 3 мкB являются наиболее мощными маркерами высокого риска возникновения НЖА и пароксизмов ФП $[10,11]$. Существование взаимосвязи показателей СУ ЭКГ предсердного комплекса и НЖА, а также возможность влияния бронхолитической терапии на эти параметры у больных хроническими обструктивными болезнями легких была отмечена ранее [7].

Полученные данные впервые демонстрируют, что препараты одного класса фуросемид и торасемид, являясь эффективными Д, оказывают неодинаковое влияние на показатели СУ ЭКГ предсердного комплекса и НЖА. В отличие от фуросемида, терапия торасемидом, не ухудшая существенно параметры СУ ЭКГ предсердного комплекса, сопровождалась снижением частоты НЖА. В многочисленных исследованиях показаны антиальдостероновые эффекты торасемида, сопровождающиеся достоверным уменьшением фиброза миокарда, параллельным улучшением его диастолических свойств и поддержанием электролитного баланса. Этими свойствами объясняется способность торасемида снижать сердечно-сосудистую смертность по сравнению с фуросемидом, что было достоверно доказано в исследованиии TORIC (Torasemide In Chronic heart failure) [12].

\section{Выводы}

Гр. больных ИБС с ХСН характеризуется выраженными нарушениями процессов деполяризации предсердий, которые проявляются неблагоприятными изменениями показателей СУ ЭКГ предсердного комплекса.

Терапия торасемидом оказывает менее выраженное отрицательное влияние на показатели СУ ЭКГ предсердного комплекса и сопровождается снижением частоты НЖА, что делает этот препарат более предпочтительным для лечения больных.

\section{Литература}

1. Беленков Ю.А., Мареев В.Ю., Агеев Ф.Т. Хроническая сердечная недостаточность. Избранные лекции по кардиологии. М.: ГЭОТАР-Медиа 2006; 432 с.

2. Bratter DC. Diuretic therapy. N Engl Med 1998; 339(6): $387-$ 95.

3. Leier CV, Meachem JA, Schaal SF. Prolonged atrial conduction. A major predisposing factor for the development of atrial flutter. Circulation 1978; 57: 213-6.

4. Жарова Е.А., Охлопкова Т.Г., Иванов Г.Г. Параметры поздних потенциалов предсердий в динамике длительного наблюдения у больных с различными формами мерцательной аритмии. Вестн аритмологии 1998; 3: 73-5.

5. Ogawa H, Fukusaki H, Ohnishi Y. Problems on evaluating signal-averaged $\mathrm{P}$ wave as a predictor of atrial fibrillation or flutter the importance of frequency range of the filter and the influence of left atrial overload. Jap Circ J 1989; 21: 534-8.

6. Иванов Г.Г., Елеуов А.У., Дворников В.Е., Моисеенко Н.И. Поздние потенциалы предсердий: электрокардиографическая основа, методы регистрации, клиническое значение. Вестник РУДН 1998; 1: 77-86.

7. Шугушев Х.Х., Гурижева М.В., Василенко В.М. Наджелудочковые аритмии и ЭКГ высокого разрешения у больных хронической обструктивной болезнью легких на фоне бронхолитической терапии. РКЖ 2010; 6: 40-5.

8. Abe Y, Fukunami M, Shimonagata T. Prediction of paroxysmal atrial fibrillation in patients with congestive heart failure: a prospective study. Circulation 1997; 29: 2612-6.

9. Сычев О.С., Заяц М.А., Романова Е.Н. и др. Выявляемость поздних потенциалов предсердий у больных с пароксизмальной и персистирующей формами фибрилляции предсердий. Укра н кардіологічний ж 2005; 2: 35-8.

10. Abe Y, Fulouiami M, Yamada T. Prediction of transition to chronic atrial fibrillation in patients with paroxysmal atrial fibrillation by signal-averaged electrocardiography: a prospective study. Circulation 1997; 27: 405-13.

11. Abe Y, Nishida T, Yamashita K, et al. Clinical study of the predicting for paroxysmal atrial fibrillation in patients with ischemic heart disease. Am J Cardiology 1999; 84: 270-7.

12. Cosin $\mathbf{J}$, Diez $\mathbf{J}$, TORIC investigators. Torasemide in chronic heart failure: results of the TORIC study. Eur $\mathbf{J}$ Heart failure 2002; 4 (4): 507-13.

Поступила 30/05-2011 